\title{
OPEN Potential of therapeutic bile acids in the treatment of neonatal Hyperbilirubinemia
}

\author{
Lori W. E. van der Schoor ${ }^{1,2}$, Henkjan J. Verkade ${ }^{1,2}$, Anna Bertolini ${ }^{1}$, Sanne de Wit ${ }^{1}$, \\ Elvira Mennillo ${ }^{3}$, Eva Rettenmeier ${ }^{3}$, André A. Weber ${ }^{3}$, Rick Havinga ${ }^{1}$, Petra Valáškováa \\ Jana Jašprová ${ }^{4}$, Dicky Struik ${ }^{1}$, Vincent W. Bloks ${ }^{1}$, Shujuan Chen ${ }^{3}$, Andrea B. Schreuder ${ }^{1,2}$, \\ Libor Vítek ${ }^{4}{ }^{4}$, Robert H. Tukey ${ }^{3 凶}$ \& Johan W. Jonker ${ }^{1 \bowtie}$
}

Neonatal hyperbilirubinemia or jaundice is associated with kernicterus, resulting in permanent neurological damage or even death. Conventional phototherapy does not prevent hyperbilirubinemia or eliminate the need for exchange transfusion. Here we investigated the potential of therapeutic bile acids ursodeoxycholic acid (UDCA) and obeticholic acid (OCA, 6- $\alpha$-ethyl-CDCA), a farnesoid-X-receptor (FXR) agonist, as preventive treatment options for neonatal hyperbilirubinemia using the $h U G T 1 * 1$ humanized mice and Ugt1a-deficient Gunn rats. Treatment of $h U G T 1 * 1$ mice with UDCA or OCA at postnatal days $10-14$ effectively decreased bilirubin in plasma (by $82 \%$ and $62 \%$ ) and brain (by $72 \%$ and $69 \%$ ), respectively. Mechanistically, our findings indicate that these effects are mediated through induction of protein levels of hUGT1A1 in the intestine, but not in liver. We further demonstrate that in Ugt1a-deficient Gunn rats, UDCA but not OCA significantly decreases plasma bilirubin, indicating that at least some of the hypobilirubinemic effects of UDCA are independent of UGT1A1. Finally, using the synthetic, non-bile acid, FXR-agonist GW4064, we show that some of these effects are mediated through direct or indirect activation of FXR. Together, our study shows that therapeutic bile acids UDCA and OCA effectively reduce both plasma and brain bilirubin, highlighting their potential in the treatment of neonatal hyperbilirubinemia.

Unconjugated bilirubin (UCB) is a potentially neurotoxic metabolite of heme catabolism. Severe unconjugated hyperbilirubinemia is associated with the development of kernicterus, resulting in permanent neurological damage or even death ${ }^{1}$. Unconjugated hyperbilirubinemia occurs primarily in newborns since they display an increased bilirubin production and a decreased disposal, caused by a combination of increased erythrocyte breakdown and very low or absent expression of the bilirubin conjugation enzyme, UDP-glucuronosyltransferase $1 \mathrm{~A} 1$ (UGT1A1) ${ }^{2}$. In newborns, bilirubin is also subject to enterohepatic circulation, resulting in substantial intestinal re-absorption and increased systemic exposure ${ }^{3}$. In preterm infants, unconjugated hyperbilirubinemia is especially problematic, since their blood-brain barrier is more permeable and their underdeveloped brain is more susceptible to bilirubin-induced neurotoxicity ${ }^{4}$.

Phototherapy enhances bilirubin disposal from the body and has been the gold standard therapy for unconjugated hyperbilirubinemia for over 60 years ${ }^{5}$. Phototherapy, however, is not always sufficiently effective in infants and does not completely eliminate the need for exchange transfusions ${ }^{6}$. Moreover, phototherapy can only decrease already accumulated UCB but does not prevent its accumulation. In epidemiological studies, phototherapy has also been associated with increased mortality and with the development of infantile cancer ${ }^{7-11}$. Thus, despite the apparent success of phototherapy, there is still a need for alternative or complementary treatments.

The bile acid (BA) ursodeoxycholic acid (UDCA) is commonly used for the treatment of cholestatic liver diseases, and has recently been shown beneficial as an adjuvant treatment for neonatal hyperbilirubinemia ${ }^{12}$. Since we and others have shown that UDCA can successfully decrease plasma bilirubin levels ${ }^{12,13}$, we hypothesized

\footnotetext{
${ }^{1}$ Section of Molecular Metabolism and Nutrition, Laboratory of Pediatrics, University of Groningen, University Medical Center Groningen, Hanzeplein 1, 9713 GZ Groningen, The Netherlands. 'Pediatric Gastroenterology and Hepatology, University of Groningen, University Medical Center, Hanzeplein 1, 9713 GZ Groningen, The Netherlands. ${ }^{3}$ Laboratory of Environmental Toxicology, Department of Pharmacology, University of California, San Diego, La Jolla, CA 92093, USA. ${ }^{4}$ Fourth Department of Internal Medicine and Institute of Medical Biochemistry and Laboratory Diagnostics, First Faculty of Medicine, Charles University, Prague, Czech Republic. ${ }^{\square}$ email: rtukey@ucsd.edu; j.w.jonker@umcg.nl
} 
a

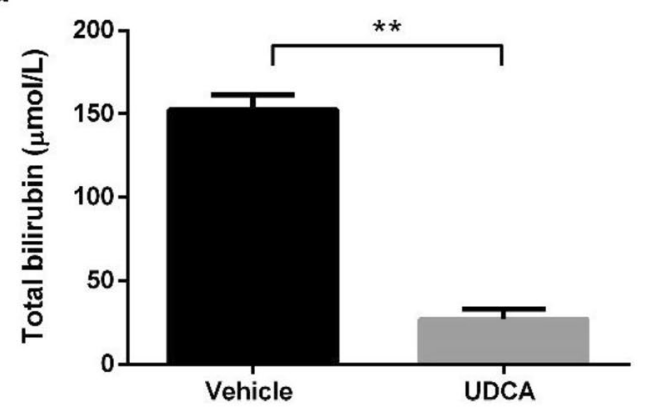

b

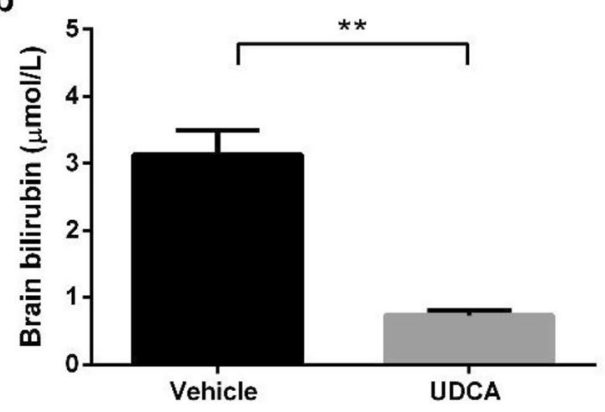

C

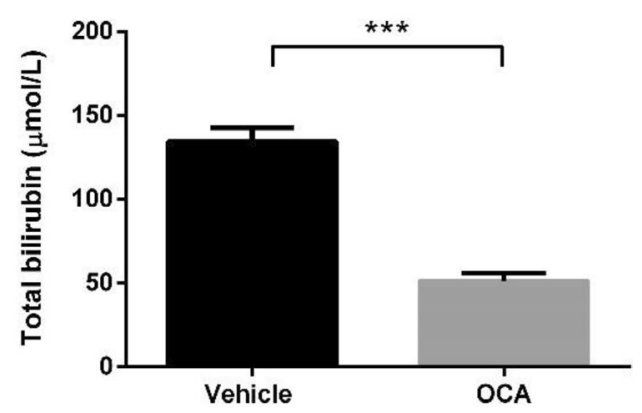

d

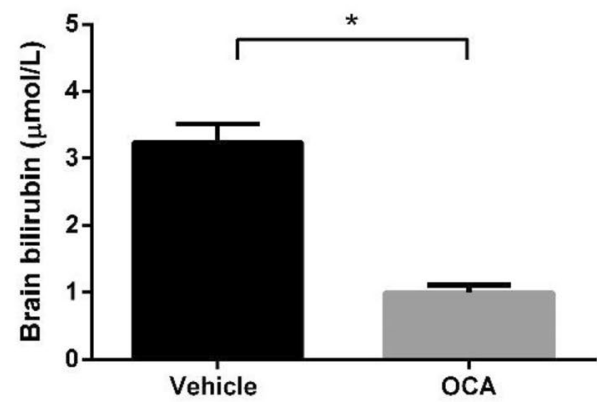

Figure 1. Effect of UDCA and OCA on plasma and brain bilirubin concentrations in neonatal hUGT1*1 mice. Levels of plasma and brain bilirubin in neonatal in hUGT1 ${ }^{\star} 1$ mice after treatment with (a, b) UDCA (250 mg/ $\mathrm{kg} /$ day, $\mathrm{n}=6)$ or $(\mathbf{c}, \mathbf{d})$ OCA $(50 \mathrm{mg} / \mathrm{kg} /$ day, $\mathrm{n}=8)$. Concentrations of bilirubin in the brain tissue are expressed as $\mu \mathrm{mol} / \mathrm{L}$ of tissue homogenate.

that other anti-cholestatic medications could also be beneficial for unconjugated hyperbilirubinemia. In 2016, obeticholic acid (OCA) was FDA-approved as a treatment for primary biliary cholangitis, for patients in which UDCA does not provide sufficient relief. OCA is a selective agonist of the farnesoid-X-receptor (FXR, NR1H4), a biological sensor for BAs that, among other functions, controls BA homeostasis by modulation of their synthesis and enterohepatic transport ${ }^{14,15}$. In contrast to OCA, UDCA is not a ligand for FXR ${ }^{16}$.

In this study, we used the humanized $h U G T 1^{\star} 1$ mouse model for unconjugated hyperbilirubinemia. $h U G T 1^{\star} 1$ mice are transgenic for the entire human UGT1A locus, including the UGT1A1 promoter and the well-described phenobarbital response enhancing module (PBREM $)^{17}$. Because UGT1A1 is expressed at an earlier age in mice as compared to humans, neonatal hyperbilirubinemia normally does not occur in mice ${ }^{18,19}$. Humanized $U G T 1^{\star} 1$ mice, however, display a similar transcriptional delay in expression of UGT1A1, resulting in severe unconjugated hyperbilirubinemia during the first three weeks of life. After three weeks, the bilirubin levels decrease upon UGT1A1 expression and the mice remain normobilirubinemic during adulthood ${ }^{17}$. Complete knockout of the Ugt1 locus in mice, when not rescued with expression of the human UGT1 locus, results in death within $4-11$ days after birth ${ }^{17,20}$. The expression of the human $U G T 1$ locus in $h U G T 1^{\star} 1$ mice rescues the lethality associated with severe neonatal hyperbilirubinemia as a result of the extrahepatic intestinal expression of the human UGT1A1 gene ${ }^{17,21}$. The physiological inducibility of $h U G T 1 A 1$ expression in $h U G T 1^{\star} 1$ mice in both liver and intestine makes this an attractive model and superior to Ugt1 deficient models such as the Ugt1a knockout mice or Gunn rats, commonly used models for hyperbilirubinemia ${ }^{22,23}$. This inducibility of $h U G T 1 A 1$ makes them similar to human neonates, and the only model to study the effects of drugs on human UGT1A1 expression and bilirubin conjugation. In this study, we assessed the effects of UDCA and OCA on bilirubin levels in plasma and brain in neonatal $h U G T 1^{\star} 1$ mice. In addition, Ugt1a deficient Gunn rats were used as a model to distinguish between UGT1A1-dependent and independent treatment effects.

\section{Results}

Effect of UDCA and OCA on plasma and brain levels of bilirubin in $h U G T 1 * 1$ mice. To determine the potential of anti-cholestatic drugs for the treatment of neonatal hyperbilirubinemia, $h U G T 1^{\star} 1$ pups were treated for five days (from P10-14) with UDCA $(\mathrm{n}=6)$ or OCA $(\mathrm{n}=8)$. UDCA $(250 \mathrm{mg} / \mathrm{kg} /$ day $)$ decreased total plasma bilirubin (TPB) by $82 \%$ (from 152 to $27 \mu \mathrm{mol} / \mathrm{L}, \mathrm{p}<0.01$ ), whereas OCA $(50 \mathrm{mg} / \mathrm{kg} /$ day) caused a $62 \%$ decrease (from 134 to $51 \mu \mathrm{mol} / \mathrm{L}, \mathrm{p}<0.001$ ). Both UDCA and OCA also significantly decreased brain bilirubin concentrations by $77 \%$ (from 3.1 to $0.7 \mu \mathrm{mol} / \mathrm{L}, \mathrm{p}<0.01$ ) and $69 \%$ (from 3.2 to $1.0 \mu \mathrm{mol} / \mathrm{L}, \mathrm{p}<0.03$ ), respectively (Fig. 1), indicating a decreased neurotoxicity risk in these mice. The UDCA-induced TPB decrease was already apparent at $30 \mathrm{mg} / \mathrm{kg} /$ day $(9 \%, \mathrm{p}=0.02)(\mathrm{n}=6)$, which is in the clinically used dose range of UDCA for preterm 
mRNA

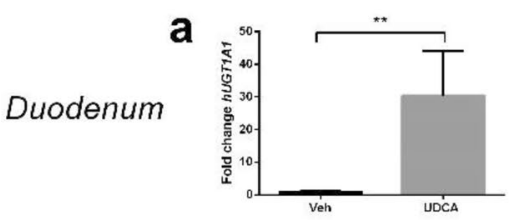

C

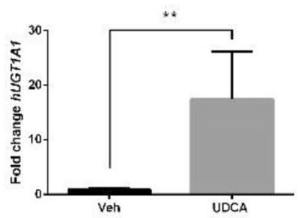

e

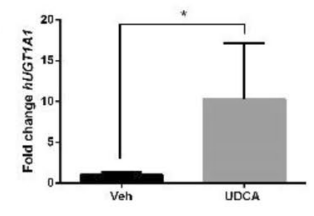

Ileum

Jejunum

g

Colon b

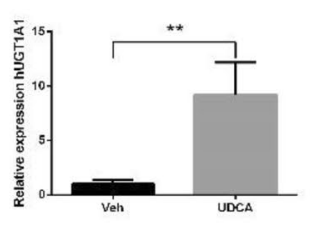

d

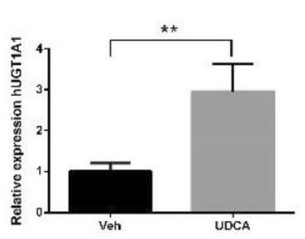

f

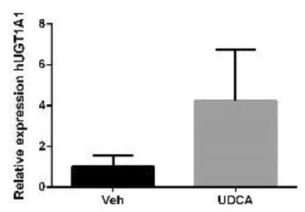

h

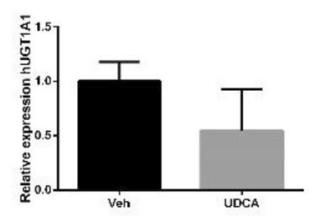

i

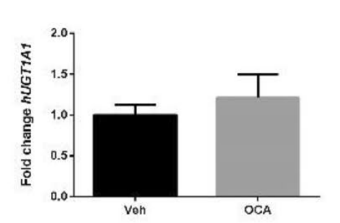

k

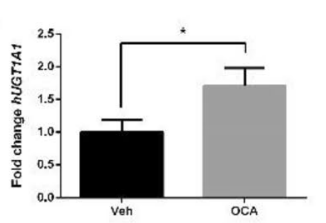

m

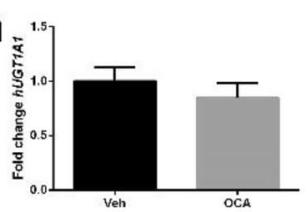

$\mathbf{0}$

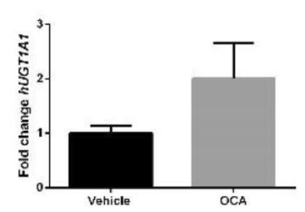

mRNA

protein

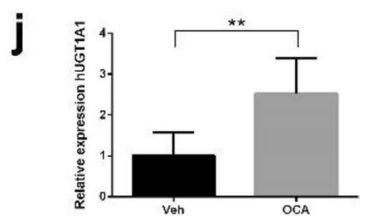

I

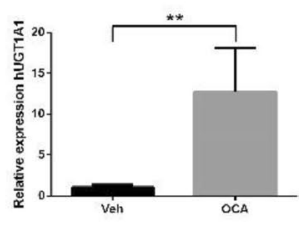

n

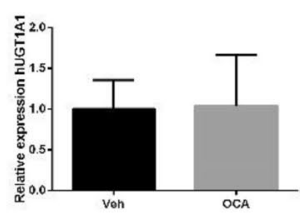

p

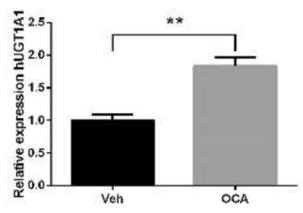

Figure 2. Effect of UDCA and OCA on intestinal hUGT1A1 mRNA and protein expression in neonatal hUGT1 ${ }^{\star} 1$ mice. hUGT1A1 gene and protein expression after UDCA treatment in $(\mathbf{a}, \mathbf{b})$ duodenum, $(\mathbf{c}, \mathbf{d})$ jejunum, $(\mathbf{e}, \mathbf{f})$ ileum and $(\mathbf{g}, \mathbf{h})$ colon $(\mathbf{n}=6)$. hUGT1A1 expression after OCA treatment in $(\mathbf{i}, \mathbf{j})$ duodenum, $(\mathbf{k}$, l) jejunum, $(\mathbf{m}, \mathbf{n})$ ileum and $(\mathbf{o}, \mathbf{p})$ colon $(n=8)$.

neonates. This decrease in TPB was also associated with decreased brain bilirubin content (18\%), although this did not reach statistical significance (Fig. S1).

Effect of UDCA and OCA on hUGT1A1 expression in liver and intestine. To assess whether UDCA or OCA affected UGT1A1 expression in $h U G T 1^{*} 1$ neonatal mice, we determined mRNA and protein levels in liver and intestine. Classically, the liver is regarded as the main organ responsible for bilirubin conjugation ${ }^{24}$. As expected, UGT1A1 expression was not detectable in the liver of neonatal $h U G T 1^{*} 1 \mathrm{mice}^{17}$, and was also not induced by UDCA or OCA (data not shown). In contrast to the liver, UDCA treatment strongly increased levels of UGT1A1 mRNA in all intestinal sections (duodenum, jejunum, ileum and colon; Fig. 2a,c,e,g), whereas OCA treatment (Fig. 2i,k,m,o) only caused a 1.7 -fold induction in jejunum $(\mathrm{p}<0.05)$ (Fig. $2 \mathrm{k}$ ). To assess whether the increased intestinal UGT1A1 gene expression also resulted in increased protein expression, we performed Western blot analysis. In line with its mRNA levels, UGT1A1 protein levels were undetectable in liver whereas both UDCA (Figs. 2b,d,f,h; Fig. S2) and OCA (Figs. 2j,l,n,p; Fig. S2) treatment significantly increased hUGT1A1 protein levels in duodenum (both $\mathrm{p}<0.01$ ) and jejunum (both $\mathrm{p}<0.01$ ), but not in ileum. In colon, UGT1A1 protein expression was only induced after OCA treatment $(\mathrm{p}<0.01)$ (Fig. $2 \mathrm{p})$.

FXR activation by UDCA and OCA. OCA is a selective FXR agonist, and therefore its actions can be potentially explained by activation of FXR. UDCA, on the other hand, is not an FXR agonist, but since it modulates BA homeostasis, it is possible that it can still indirectly affect FXR activity. To determine whether OCA and UDCA (directly or indirectly) caused FXR activation, we determined mRNA levels of the established FXR targets short heterodimer partner (Shp, Nrob2), cholesterol 7a-hydroxylase (Cyp7a1) and the bile salt exporting protein (Bsep, Abcb11) in liver and of Shp and fibroblast growth factor 15 (Fgf15) in ileum (Fig. 3). Both UDCA and OCA treatment resulted in hepatic and ileal FXR activation, although Bsep expression was not significantly affected by UDCA (Fig. 3). 
$U D C A$

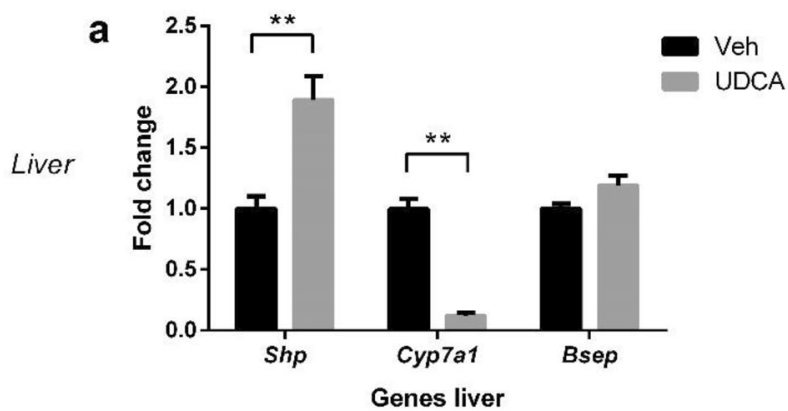

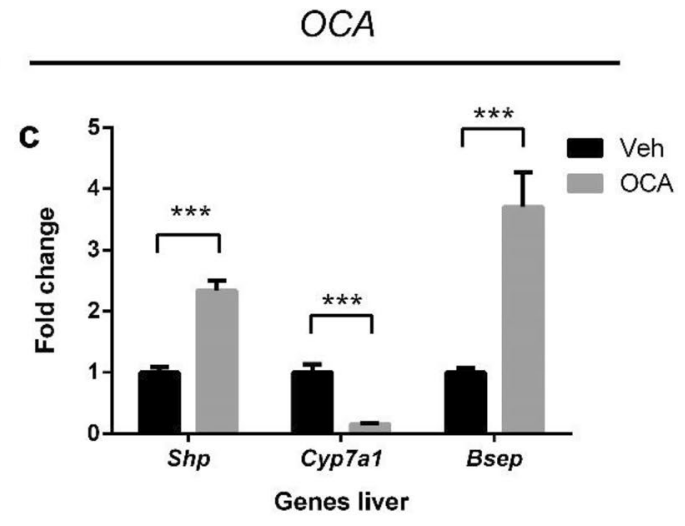

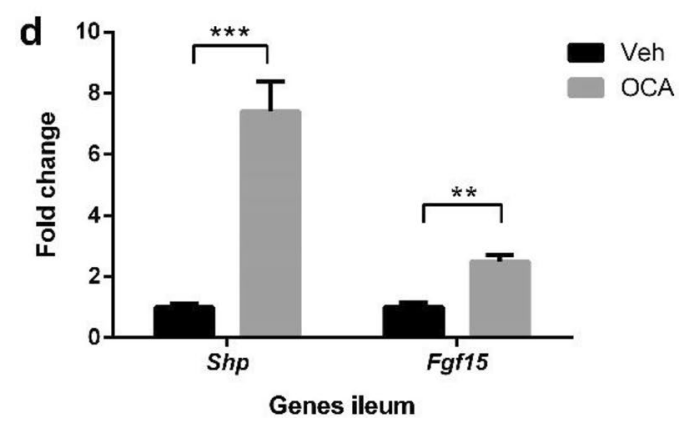

Figure 3. Effect of UDCA and OCA on expression of FXR-target genes in neonatal hUGT1 ${ }^{\star} 1$ mice. mRNA levels of (a) hepatic and (b) ileal FXR target genes after vehicle or UDCA; (c) hepatic and (d) ileal FXR target genes after vehicle or OCA.

Based on these findings, we hypothesized that the effects on UCB levels in plasma and brain could be mediated through FXR activation, either directly (OCA) or indirectly (UDCA). To further evaluate the potential involvement of FXR, we also tested the UCB lowering effects of the synthetic, non-BA, FXR-agonist GW4064, which is known to decrease BA production by suppression of CYP7A1. Treatment of $h U G T 1^{\star} 1$ pups with GW4064 $(50 \mathrm{mg} / \mathrm{kg} /$ day $)(\mathrm{n}=6)$ resulted in a similar gene expression pattern matching with FXR activation (Fig. S3). GW4064 also significantly reduced TPB by $13 \%(\mathrm{p}<0.05)$ and brain UCB by $31 \%(\mathrm{p}<0.01)$ (Fig. 4a,b), along with a twofold induction of UGT1A1 protein expression in duodenum (Fig. 4d). GW4064 also significantly increased UGT1A1 mRNA expression in ileum, but this was not accompanied by increased protein expression (Fig. 4g,h). In jejunum and colon, GW4064 did not induce UGT1A1 (Fig. 4e,f,i,j). Collectively, these observations support a role for FXR in the UCB lowering effects of tested anti-cholestatic drugs.

Contribution of UGT1A1 to the bilirubin-lowering effects of UDCA and OCA. Although $h U G T 1^{\star} 1$ mice are dependent on hUGT1A1 expression for survival, this does not mean that the UDCA- and OCA-induced effects in these mice are necessarily or exclusively UGT1A1-mediated. To assess the role of UGT1A1, we therefore also tested both compounds in the Ugt1a-deficient Gunn rat model. In line with our previous observations in adult Gunn rats ${ }^{13}$, UDCA caused a significant decrease in plasma bilirubin levels of neonatal Gunn rats (26\%, $\mathrm{p}<0.05)$ whereas no effect was observed on brain bilirubin levels. In contrast, both OCA and GW4064 did not affect plasma or brain bilirubin levels in neonatal Gunn rats (Fig. 5). Unlike OCA and GW4064, UDCA treatment did not activate FXR in liver and intestine, indicating that the hypobilirubinemic effects of UDCA in Gunn rats were independent of FXR activation (Fig. S4).

\section{Discussion}

In this study, we show that the therapeutic BAs UDCA and OCA effectively decrease plasma bilirubin in a humanized mouse model of neonatal hyperbilirubinemia. Both compounds also significantly reduced brain levels of bilirubin in these mice, highlighting their potential in the prevention of bilirubin-induced neurotoxicity. Mechanistically, we demonstrated that these effects are at least partially dependent on induction of intestinal UGT1A1 expression and on FXR activation.

Neonatal hyperbilirubinemia is caused by a limited capacity to metabolize bilirubin mainly due to delayed expression of UGT1A1. Since rodents express UGT1A1 at an earlier age than humans, they normally do not exhibit neonatal hyperbilirubinemia. For mice, it is known that a complete deficiency in UGT1A1 expression is lethal within the first $4-7$ postnatal days ${ }^{17,20}$, and that this condition can be rescued by bilirubin lowering treatments such as intraperitoneal albumin administration, Ugt1a1 replacement gene therapy or by transgenic introduction of the human UGT1A1 gene and its upstream regulatory region ${ }^{20,25}$. In $h U G T 1^{\star} 1$ mice, $U G T 1 A 1$ expression in neonates is almost exclusively intestinal, whereas in adults, it is expressed both in intestine and 


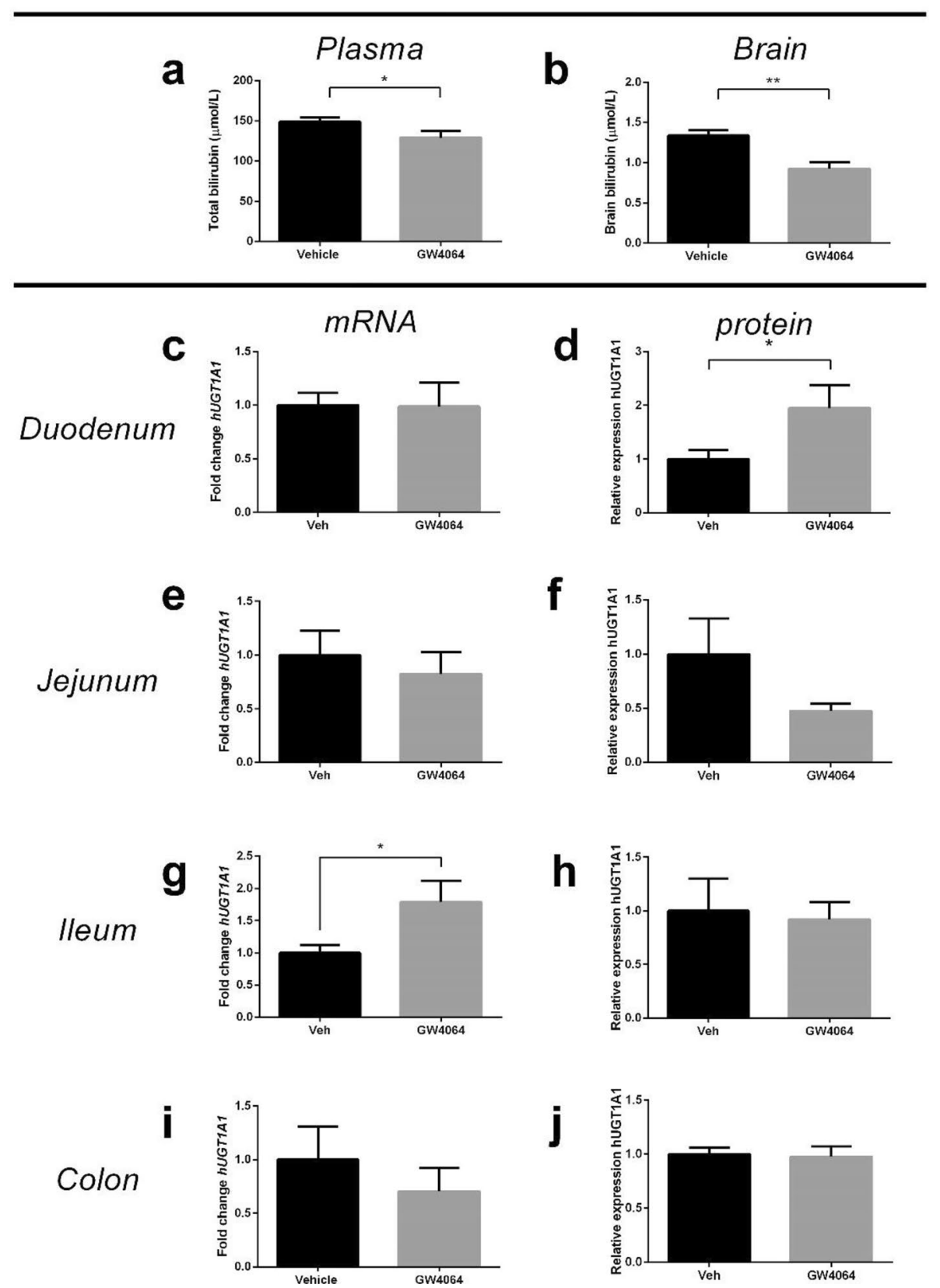

Figure 4. Effect of GW4064 on plasma and brain bilirubin and intestinal expression of hUGT1A1 in neonatal hUGT1 ${ }^{\star} 1$ mice. Effect of GW4064 treatment $(50 \mathrm{mg} / \mathrm{kg} /$ day $)(\mathrm{n}=6)$ on (a) Total plasma bilirubin level; (b) Brain bilirubin levels; (c, d) mRNA and protein expression of hUGT1A1 in duodenum; and mRNA and protein expression of hUGT1A1 in (e, f) jejunum; $(\mathbf{g}, \mathbf{h})$ ileum and $(\mathbf{i}, \mathbf{j})$ colon.

liver ${ }^{17}$. In human neonates, the intestinal UGT1A1 expression and its potential contribution to neonatal bilirubin metabolism have never been studied, but there are several indications supporting the concept that intestinal bilirubin conjugation is also important in human physiology. Besides the liver, UGT1A1 is also highly expressed in the small intestine. Already in the ' $90 \mathrm{~s}$, McDonnel et al. demonstrated UGT1A1 expression and bilirubin conjugation activity in the human intestinal tract ${ }^{26}$. In adult human tissues, the intestinal conjugation of the UGT1A1 substrate estradiol even exceeds the hepatic conjugation capacity ${ }^{27}$. In premature human newborns, UGT1A1 gene expression is repressed in the liver during the first postnatal days ${ }^{18,19}$. It is likely that intestinal UGT1A1 expression is similar between $h U G T 1 A 1$ mice and human newborns with intestinal UGT1A1 participating in the metabolism of serum bilirubin. However, this will be hard to establish both experimentally and ethically, in particular because healthy neonatal intestinal tissue is hardly available. 
$U D C A$

a

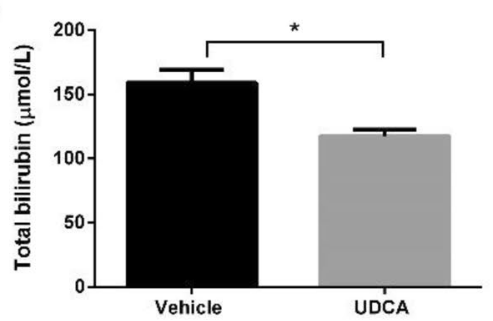

b

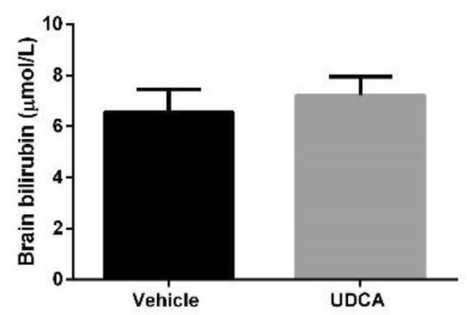

OCA

C

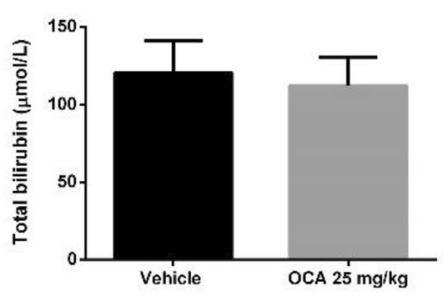

d

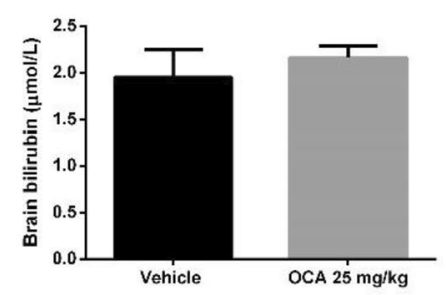

GW4064

e

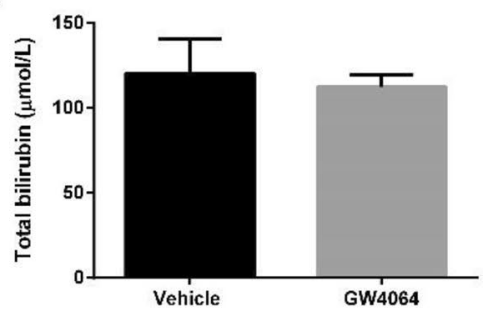

f

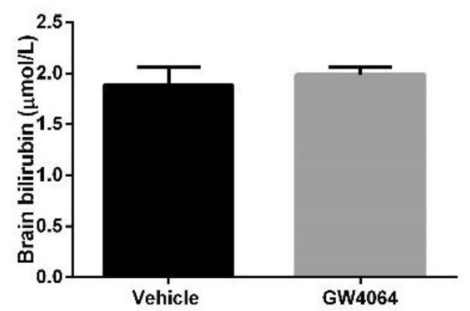

Figure 5. Effect of UDCA, OCA, and GW4064 on plasma and brain bilirubin in neonatal Gunn rats. Effect of (a, b) UDCA (250 mg/kg/day) (n=6); (c, d) OCA (25 mg/kg/day) $(\mathrm{n}=8)$, and (e, f) GW4064 (50 mg/kg/day) $(n=6)$ on total bilirubin levels in plasma and brain.

Both UDCA and OCA induced UGT1A1 mRNA and protein expression in duodenum and jejunum, which are also intestinal expression sites in human ${ }^{26,28,29}$. This induction could potentially be FXR-mediated, as we observed a significant bilirubin lowering effect by both OCA and GW4064, which are two structurally unrelated FXR ligands. Despite this, the bilirubin lowering effect by GW4064 and its induction of intestinal Ugt1a1 were less prominent as compared to OCA. These differences are most likely explained by their different chemical structures and pharmacokinetic profiles. While OCA is a bile acid analogue which exhibits bile acid like pharmacokinetics (enterohepatic cycling), GW4064 is a non-steroidal (non-bile acid) synthetic FXR ligand with a relatively poor oral availability and which does not undergo enterohepatic cycling. These characteristics might result in a lower induction of intestinal Ugt1a1 by GW4064.

Alternatively, the induction could be indirectly regulated, for example through activation of the pregnane-Xreceptor (PXR, NR1I2), another member of the nuclear receptor superfamily. PXR activation results in a robust transcriptional induction of the UGT1A1 gene while PXR itself can also be activated by BAs and transcriptionally regulated by $\mathrm{FXR}^{30,31}$. Besides regulation at the transcriptional level, it is also possible that hUGT1A1 induction is (partially) mediated at the posttranslational level. OCA, for example, did not increase mRNA levels of hUGT1A1 in duodenum, but did induce the protein expression by 2.5 -fold, which can potentially be explained by increased protein stability. Finally, FXR-independent mechanisms could play a role in the induction of hUGT1A1 by UDCA and OCA. To investigate this, however, it would require an Fxr-deficient humanized $U G T 1^{\star} 1$ mouse model, which is currently not available.

Our work also indicates that UDCA acts independently of UGT1A1, as UDCA treatment decreased TPB in Ugt1a1-deficient Gunn rats. This finding is in line with our previous studies in adult Gunn rats, in which UDCA decreased TPB by $21 \%$ after 7 days of treatment ${ }^{13}$. The TPB reduction in Gunn rat pups (-26\%) is lower than the reduction observed in $h U G T 1^{\star} 1$ pups $(-86 \%)$ and this can potentially be explained by a cumulative effect of UGT1A1-dependent and -independent UDCA effects in the latter.

There are several possible explanations for the hUGT1A1-independent effects. Upon treatment, UDCA becomes the major component of the BA pool, and thereby affects both the hepatic and intestinal BA composition $^{13}$. Changes in intestinal BA composition could cause alterations in both hepatic and intestinal bilirubin metabolism. Hepatic conjugation is not possible in Gunn rats, and in previous work by our group, UDCA did not increase the biliary bilirubin concentration ${ }^{13}$. This makes hepatic interference of UDCA in bilirubin metabolism unlikely. However, in the same study, it was shown that UDCA caused a significant increase in fecal bilirubin, which indicates that UDCA either diminishes the intestinal bilirubin reabsorption or stimulates direct intestinal bilirubin excretion. Possibly, these mechanisms also play a role in the neonatal $h U G T 1^{\star} 1$ mice, but this could not be tested in the current study since collection of sufficient bile or feces was impossible at this young age.

The fact that OCA and GW4064 did not reduce TPB in Gunn rats, in contrast to $h U G T 1^{\star} 1$ mice, indicates that $h U G T 1 A 1$ expression is essential for the bilirubin-lowering effect. Another possible explanation for the absent effect of OCA could be the lower OCA dose used in rats $(25 / \mathrm{mg} / \mathrm{kg} /$ day) compared to mice $(50 \mathrm{mg} / \mathrm{kg} /$ day). This approach was chosen because the high OCA dose was poorly tolerated in rats (data not shown). This lower dose could be responsible for the absent OCA effect, but not for the lack of effect by GW4064, which was administered at the same dose to Gunn rats and $h U G T 1^{\star} 1$ mice.

Although adult Gunn rats have been widely used to study neonatal hyperbilirubinemia, neonatal $h U G T 1^{\star} 1$ mice appear to be a more relevant model, since like human neonates, they have inducible hUGT1A1 expression 
that mediates the postnatal bilirubin course. Furthermore, it is a strictly neonatal model, which more closely mimics the human neonatal physiologic situation with a yet underdeveloped intestine, that is solely exposed to milk feeding. In adult humans and wild-type rodents, bilirubin levels barely depend on intestinal bilirubin metabolism and reabsorption, since in the presence of a fully-developed intestinal microbiome, bilirubin is effectively converted to urobilinogen and other derivates ${ }^{32,33}$. These metabolites are either not reabsorbed by the intestine, or efficiently excreted via the urine or bile after intestinal reabsorption ${ }^{34}$. However, in an inadequately colonized intestine, as typical for early neonatal period or after oral antibiotic treatment ${ }^{32}$, intestinal reabsorption profoundly contributes to the high TBP level ${ }^{35,36}$. Therefore, it will be interesting to target intestinal bilirubin metabolism as a treatment strategy, especially in neonatal hyperbilirubinemia. UDCA and OCA alter the intestinal milieu and could thereby affect intestinal bilirubin handling, which could be especially beneficial during the neonatal period.

The UGT1A1 gene in neonatal $h U G T 1^{\star} 1$ mice is transcriptionally regulated by nuclear receptors PXR, the constitutive-androstane receptor (CAR, NR1I3), and peroxisome proliferator-activated receptor $\alpha$ (PPAR $\alpha)^{30,37,38}$. Induction of UGT1A1 was one of the underlying mechanisms of treatment with phenobarbital, a CAR agonist used as a standard treatment for neonatal hyperbilirubinemia before the introduction of phototherapy ${ }^{39,40}$ and used in Crigler-Najjar syndrome type $2^{41}$. hUGT1 mice do not only contain the UGT1A1 gene, but also the upstream regulatory region, including the distal regulatory PBREM that is activated by PXR and CAR ${ }^{37}$. This is important, since the $h U G T 1 A 1$ promoter regions are not conserved between species and transcriptional regulation of the gene differs between rodents and humans. By merely studying potential therapies in Ugt1a1-deficient models, such as Gunn rats, clinical effects could be missed, as we show for OCA, which did not decrease bilirubin in Gunn rats.

Bilirubin neurotoxicity is caused by brain bilirubin deposition (kernicterus) and therefore, strongly depends on the maturity and permeability of the blood-brain barrier ${ }^{36}$. In homozygous and heterozygous Gunn rats, we observed that brain bilirubin levels are significantly higher in neonates as compared to (young) adults and that the ratio between brain and plasma bilirubin is higher in neonates than in adults (data not shown). This phenomenon has also been described in piglets, in which the ratio between brain bilirubin and free bilirubin in plasma decreases fivefold between $\mathrm{P} 2$ and $\mathrm{P} 14^{42}$. Therefore, we used neonatal animals to study the effects of UDCA and OCA on brain bilirubin deposition and the consequent neurotoxicity risk.

Interestingly, we show that UDCA only decreased plasma, but not brain bilirubin levels in neonatal Gunn rats, whereas it decreased both plasma and brain bilirubin levels in neonatal $h U G T 1^{\star} 1$ mice. This phenomenon could have several explanations. Firstly, a more profound decrease in plasma bilirubin levels might be needed to induce a decrease in brain bilirubin in the Gunn rat. However, in the $h U G T 1^{\star} 1$ mice, the relative decrease in brain bilirubin seemed to follow closely the corresponding decrease in plasma. This possibly results from the fact that $U G T 1 A 1$ is expressed in brain of $h U G T 1^{\star} 1$ mice. Although bilirubin conjugation in the brain has never been demonstrated, UGT1A1 was previously shown to mediate estradiol conjugation in the brain ${ }^{43}$. Therefore, brain UGT1A1 could potentially contribute to local bilirubin biotransformation and export. Secondly, bilirubin is exported from the brain by species-specific transporters such as Mdr1a P-glycoprotein (Abcb1a), which are differentially regulated between mice and rats ${ }^{44}$. Potentially, UDCA may upregulate a mouse transport protein that is not present or not regulated similarly in Gunn rats.

In conclusion, we show that both UDCA and OCA strongly decrease bilirubin in plasma and brain of neonatal $h U G T 1^{\star} 1$ mice. Mechanistically, our work indicates that intestinal, rather than hepatic hUGT1A1, plays an important role in these effects. Furthermore, it suggests that OCA acts via hUGT1A1-dependent mechanisms, whereas UDCA also has partial hUGT1A1-independent effects. Both UDCA and OCA are FDA-approved drugs, and UDCA is even already approved for neonatal use, making them readily available for clinical studies. Both compounds could potentially offer a prevention strategy for neonatal hyperbilirubinemia.

\section{Methods}

Animals. The generation of humanized $U G T 1^{\star} 1$ mice (>99\% C57BL/6J background) has been described previously ${ }^{17}$. Before and during the experiments, all pups were housed with their mother with ad libitum access to breast milk. Dams had ad libitum access to water and food (Picolab Rodent Diet 20, 5053 breeding diet, (Labdiet, St. Louis, MO). The litters were kept in an environmentally controlled facility with a $12 \mathrm{~h}$ day/night cycle. Gunn rats (originally obtained from Rat Resource Center (Columbia, MO) were bred at the University Medical Center Groningen (UMCG) animal facility. Dams were fed ad libitum with RM03 breeding chow (Special Diet Services, Essex, UK).

Animal procedures. Pups (both male and female) within every litter were randomized to receive vehicle (ORA-plus oral suspension solution, Allegan, MI) or vehicle with either UDCA (Sigma-Aldrich, St. Louis, MO), OCA (MedChemExpress, South Brunswick, NJ), or GW4064 (Cayman Chemicals, Ann Arbor, MI). UDCA was given at $250 \mathrm{mg} / \mathrm{kg} /$ day once daily via oral gavage. Both OCA and GW4064 were given at a dose of $50 \mathrm{mg} / \mathrm{kg} /$ day, divided over 2 doses daily. Also, $h U G T 1^{\star} 1$ mice were treated with the clinically recommended UDCA dose for neonates $(30 \mathrm{mg} / \mathrm{kg} /$ day in 2 doses per day). All mice were treated daily from P10-14. Mice were sacrificed within $6 \mathrm{~h}$ after the last dose on P14 by decapitation under isoflurane anesthesia. Blood was collected in EDTAcoated capillaries from the trunk and stored on ice in the dark upon centrifugation and plasma was stored at $-80^{\circ}$ $\mathrm{C}$ under argon until analysis. Liver and spleen were snap-frozen upon harvesting and subsequently pulverized. The intestine was flushed with ice-cold PBS before snap freezing. Brains were snap frozen and stored in ambercolored tubes at $-80^{\circ} \mathrm{C}$. Since OCA is associated with hepatotoxicity, we tested its effect on plasma activities of ALT and AST. OCA treatment induced moderately increased activities of plasma ALT but had no significant effect on plasma AST, suggesting that hepatotoxicity under these conditions was limited (Fig. S5). 
Gunn rat pups were treated with UDCA ( $250 \mathrm{mg} / \mathrm{kg} /$ day) divided over 2 daily doses from P7-14 or with OCA (25 mg/kg/day) or GW4064 (50 mg/kg/day) from P10-14 in one daily dose. Gunn rat pups were terminated on $\mathrm{P} 14$ within $6 \mathrm{~h}$ after the last dose by cardiac puncture under isoflurane anesthesia and tissues collected and stored as described. All experiments with $h U G T 1^{\star} 1$ mice were performed at UCSD and approved by the local Animal Ethics Committee of University of California San Diego. All experiments with Gunn rats were performed at the UMCG with the approval of the local Ethics Committee for Animal Experiments of the University of Groningen. All experiments were performed in accordance with relevant guidelines and regulations (including laboratory and biosafety regulations).

Bilirubin analysis. TPB analysis in $h U G T 1^{\star} 1$ mice was performed using a UNISTAT Bilirubinometer (Reichert Technologies, Depew, NY). TPB analysis in Gunn rats was performed using the Bilirubin Total Gen 3 kit (Roche Diagnostics, Rotkreuz, Switzerland) on a Roche/Hitachi Cobas 501 Analyzer (Hitachi, Tokyo, Japan). Analysis of bilirubin in the brain tissue was performed as described previously ${ }^{45}$. Briefly, approximately $100 \mathrm{mg}$ of homogenized brain was mixed with $50 \mu \mathrm{L}$ of $5 \mu \mathrm{M}$ internal standard mesobilirubin (Frontier Scientific, Logan, UT) and homogenized with glass rod. Bilirubin from this mixture was extracted into methanol/chloroform/ hexane $(10 / 5 / 1, \mathrm{v} / \mathrm{v} / \mathrm{v})$. Afterwards, the resulting phase was mixed with $\mathrm{n}$-hexane and carbonate buffer $(\mathrm{pH}$ $10)$, vortex-mixed and centrifuged. Fifty $\mu \mathrm{L}$ of the resulting polar droplet was loaded onto reverse C- 8 column (Luna $3 \mu \mathrm{m}, 150 \times 4.6 \mathrm{~mm}$, Phenomenex, Torrance, CA) and the amount of bilirubin was determined on HPLC (Agilent 1200 DAD, Agilent, Santa Clara, CA). Concentrations of bilirubin in the brain tissue were expressed in $\mu \mathrm{mol} / \mathrm{L}$ of tissue homogenate.

Gene expression analysis. Total RNA was isolated from the liver, small intestine (duodenum, jejunum, ileum) and colon of mice using Trizol (Life Technologies, USA) and reverse transcribed into cDNA using iSCRIPT (Bio-Rad). For quantitative PCR (qPCR), cDNA was amplified using Hi-ROX SensiMix ${ }^{\text {tw }}$ SYBR green (Bioline, London, UK) or Taqman fast mix (Applied Biosystems, Foster City, CA) using the Quant Studio RealTime qPCR (Applied Biosystems) with gene-specific primers (Table S1). Each sample was quantified using a standard curve of the pooled samples and normalized for cyclophilin.

Protein analysis. For immunoblot analysis, tissue lysates were obtained using RIPA lysis buffer (ThermoFischer Scientifc, Waltham, MA). After determination of protein concentration, $30 \mu \mathrm{g}$ protein was loaded on a pre-cast NuPAGE BisTris gel (Thermofisher, Waltham, MA). The resolved protein was transferred onto a $45 \mu \mathrm{m}$ PVDF membrane (Merck Millipore, Burlington, MA). After blocking, the membrane was incubation with hUGT1A1 primary antibody (ab170858, Abcam, Cambridge, UK) and GAPDH (sc-32233, Santa Cruz Biotechnology, Dallas, TX) and secondary antibody (anti-mouse IgG HRP-linked 7076S and anti-rabbit IgG HRPlinked 7074, Cell Signaling, Danvers, MA) detected by the Clarity Western ECL Substrate (Biorad, Hercules, CA) and visualized using the Bio-Rad ChemiDoc imaging system.

Statistical analysis. GraphPad Prism 5.00 software package (GraphPad Software, San Diego, CA) was used to perform statistical analysis. Since the that the data was not normally distributed, significance was determined using nonparametric Mann Whitney U-test. All values are given as means \pm standard error unless stated otherwise. Significance is indicated as ${ }^{\star} P<0.05,{ }^{* *} P<0.01,{ }^{* *} P<0.001$.

\section{Data availability}

The data generated in the current study are available from the corresponding author upon request.

Received: 27 August 2019; Accepted: 9 May 2021

Published online: 27 May 2021

\section{References}

1. Watchko, J. F. \& Tiribelli, C. Bilirubin-induced neurologic damage: Mechanisms and management approaches. N. Engl. J. Med. 369, 2021-2030 (2013).

2. Dennery, P. A., Seidman, D. S. \& Stevenson, D. K. Neonatal hyperbilirubinemia. N. Engl. J. Med. 344, 581-590 (2001).

3. Poland, R. L. \& Odell, G. B. Physiologic jaundice: The enterohepatic circulation of bilirubin. N. Engl. J. Med. 284, 1-6 (1971).

4. Watchko, J. F. \& Maisels, M. J. Jaundice in low birthweight infants: Pathobiology and outcome. Arch. Dis. Child. Fetal Neonatal Ed. 88, F455-F458 (2003).

5. Cremer, R. J., Perryman, P. \& Richards, D. Influence of light on the hyperbilirubinaemia of infants. The Lancet 271, 1094-1097 (1958).

6. Brown, A. K., Kim, M. H., Wu, P. Y. \& Bryla, D. A. Efficacy of phototherapy in prevention and management of neonatal hyperbilirubinemia. Pediatrics 75, 393-400 (1985).

7. Morris, B. H. et al. Aggressive vs. conservative phototherapy for infants with extremely low birth weight. N. Engl. J. Med. 359, 1885-1896 (2008).

8. Tyson, J. E. et al. Does aggressive phototherapy increase mortality while decreasing profound impairment among the smallest and sickest newborns?. J. Perinatol. 32, 677-684 (2012).

9. Arnold, C., Pedroza, C. \& Tyson, J. E. Phototherapy in ELBW newborns: Does it work? Is it safe? The evidence from randomized clinical trials. Semin. Perinatol. 38, 452-464 (2014).

10. Wickremasinghe, A. C., Kuzniewicz, M. W., Grimes, B. A., McCulloch, C. E. \& Newman, T. B. Neonatal phototherapy and infantile cancer. Pediatrics 137, 1353. https://doi.org/10.1542/peds.2015-1353 (2016).

11. Auger, N., Laverdière, C., Ayoub, A., Lo, E. \& Luu, T. M. Neonatal phototherapy and future risk of childhood cancer. Int. J. Cancer. https://doi.org/10.1002/ijc.32158 (2019).

12. Honar, N. et al. Effect of ursodeoxycholic acid on indirect hyperbilirubinemia in neonates treated with phototherapy. J. Pediatr. Gastroenterol. Nutr. 62, 97-100 (2016). 
13. Cuperus, F. J. et al. Effective treatment of unconjugated hyperbilirubinemia with oral bile salts in Gunn rats. Gastroenterology 136, 673-682 (2009).

14. Markham, A. \& Keam, S. J. Obeticholic acid: First global approval. Drugs 76, 1221-1226 (2016).

15. Gitto, S., Guarneri, V., Sartini, A. \& Andreone, P. The use of obeticholic acid for the management of non-viral liver disease: Current clinical practice and future perspectives. Exp. Rev. Gastroenterol. Hepatol. 12, 165-171 (2018).

16. Chiang, J. Y. Bile acid regulation of gene expression: Roles of nuclear hormone receptors. Endocr. Rev. 23, 443-463 (2002).

17. Fujiwara, R., Nguyen, N., Chen, S. \& Tukey, R. H. Developmental hyperbilirubinemia and CNS toxicity in mice humanized with the UDP glucuronosyltransferase 1 (UGT1) locus. Proc. Natl. Acad. Sci. USA 107, 5024-5029 (2010).

18. Kawade, N. \& Onishi, S. The prenatal and postnatal development of UDP-glucuronyltransferase activity towards bilirubin and the effect of premature birth on this activity in the human liver. Biochem. J. 196, 257-260 (1981).

19. Coughtrie, M. W., Burchell, B., Leakey, J. E. \& Hume, R. The inadequacy of perinatal glucuronidation: Immunoblot analysis of the developmental expression of individual UDP-glucuronosyltransferase isoenzymes in rat and human liver microsomes. Mol. Pharmacol. 34, 729-735 (1988).

20. Bortolussi, G. et al. Rescue of bilirubin-induced neonatal lethality in a mouse model of Crigler-Najjar syndrome type I by AAV9mediated gene transfer. FASEB J. 26, 1052-1063 (2012).

21. Chen, S. \& Tukey, R. H. Humanized UGT1 mice, regulation of UGT1A1, and the role of the intestinal tract in neonatal hyperbilirubinemia and breast milk-induced jaundice. Drug Metab. Dispos. 46, 1745-1755 (2018).

22. Chen, S., Yueh, M., Evans, R. M. \& Tukey, R. H. Pregnane-x-receptor controls hepatic glucuronidation during pregnancy and neonatal development in humanized UGT1 mice. Hepatology 56, 658-667 (2012).

23. Aoshima, N., Fujie, Y., Itoh, T., Tukey, R. H. \& Fujiwara, R. Glucose induces intestinal human UDP-glucuronosyltransferase (UGT) 1 A1 to prevent neonatal hyperbilirubinemia. Sci. Rep. 4, 643 (2014).

24. Gartner, L. M., Lee, K., Vaisman, S., Lane, D. \& Zarafu, I. Development of bilirubin transport and metabolismin the newborn rhesus monkey. J. Pediatr. 90, 513-531 (1977).

25. Vodret, S. et al. Albumin administration prevents neurological damage and death in a mouse model of severe neonatal hyperbilirubinemia. Sci. Rep. 5, 16203 (2015).

26. McDonnell, W. M., Hitomi, E. \& Askari, F. K. Identification of bilirubin UDP-GTs in the human alimentary tract in accordance with the gut as a putative metabolic organ. Biochem. Pharmacol. 51, 483-488 (1996).

27. Fisher, M. B. et al. Tissue distribution and interindividual variation in human UDP-glucuronosyltransferase activity: Relationship between UGT1A1 promoter genotype and variability in a liver bank. Pharmacogen. Genom. 10, 727-739 (2000).

28. Hruz, T. et al. Genevestigator v3: A reference expression database for the meta-analysis of transcriptomes. Adv. Bioinform. 2008, 420747 (2008).

29. Akazawa, T. et al. High expression of UGT1A1/1A6 in monkey small intestine: Comparison of protein expression levels of cytochromes P450, UDP-glucuronosyltransferases, and transporters in small intestine of cynomolgus monkey and human. Mol. Pharm. 15, 127-140 (2017).

30. Sugatani, J., Sueyoshi, T., Negishi, M. \& Miwa, M. Regulation of the human UGT1A1 gene by nuclear receptors constitutive active/ androstane receptor, pregnane X receptor, and glucocorticoid receptor. Methods Enzymol. 400, 92-104 (2005).

31. Jonker, J. W., Liddle, C. \& Downes, M. FXR and PXR: Potential therapeutic targets in cholestasis. J. Steroid Biochem. Mol. Biol. 130, $147-158$ (2012).

32. Vítek, L., Zelenka, J., Zadinová, M. \& Malina, J. The impact of intestinal microflora on serum bilirubin levels. J. Hepatol. 42, 238-243 (2005).

33. Vítek, L. et al. Identification of bilirubin reduction products formed by Clostridium perfringens isolated from human neonatal fecal flora. J. Chromatogr. B 833, 149-157 (2006).

34. Midtvedt, A. C., Carlstedt-Duke, B., Norin, K. E., Saxerholt, H. \& Midtvedt, T. Development of five metabolic activities associated with the intestinal microflora of healthy infants. J. Pediatr. Gastroenterol. Nutr. 7, 559-567 (1988).

35. Vitek, L. et al. Intestinal colonization leading to fecal urobilinoid excretion may play a role in the pathogenesis of neonatal jaundice. J. Pediatr. Gastroenterol. Nutr. 30, 294-298 (2000).

36. Ostrow, J. D., Pascolo, L., Shapiro, S. M. \& Tiribelli, C. New concepts in bilirubin encephalopathy. Eur. J. Clin. Invest. 33, 988-997 (2003).

37. Chen, S. et al. Tissue-specific, inducible, and hormonal control of the human UDP-glucuronosyltransferase-1 (UGT1) locus. J. Biol. Chem. 280, 37547-37557 (2005).

38. Senekeo-Effenberger, K. et al. Expression of the human UGT1 locus in transgenic mice by 4-chloro-6-(2, 3-xylidino)-2-pyrimidinylthioacetic acid (WY-14643) and implications on drug metabolism through peroxisome proliferator-activated receptor alpha activation. Drug Metab. Dispos. 35, 419-427 (2007).

39. Zeidenberg, P., Orrenius, S. \& Ernster, L. Increase in levels of glucuronylating enzymes and associated rise in activities of mitochondrial oxidative enzymes upon phenobarbital administration in the rat. J. Cell Biol. 32, 528-531 (1967).

40. Catz, C. \& Yaffe, S. Barbiturate enhancement of bilirubin conjugation and excretion in young and adult animals. Pediatr. Res. 2, $361(1968)$

41. Ito, T. et al. Phenobarbital following phototherapy for Crigler-Najjar syndrome Type II with good fetal outcome: A case report. J. Obstet. Gynaecol. Res. 27, 33-35 (2001).

42. Lee, C., Stonestreet, B. S., Oh, W., Outerbridge, E. W. \& Cashore, W. J. Postnatal maturation of the blood-brain barrier for unbound bilirubin in newborn piglets. Brain Res. 689, 233-238 (1995).

43. Kutsuno, Y. et al. Expression of UDP-glucuronosyltransferase 1 (UGT1) and glucuronidation activity toward endogenous substances in humanized UGT1 mouse brain. Drug Metab. Dispos. 43, 1071-1076 (2015).

44. Suzuyama, N. et al. Species differences of inhibitory effects on P-glycoprotein mediated drug transport. J. Pharm. Sci. 96, 1609-1618 (2007).

45. Zelenka, J. et al. Highly sensitive method for quantitative determination of bilirubin in biological fluids and tissues. J. Chromatogr. $B$ 867, 37-42 (2008).

\section{Acknowledgements}

We thank Henk Wolters and Angelika Jurdzinski for excellent technical assistance. This work was funded by grants from The Netherlands Organization for Scientific Research (VICI Grant 016.176.640 to JWJ), European Foundation for the Study of Diabetes (award supported by EFSD/Novo Nordisk to JWJ, Grant no. NN2018_4), a KNAW Ter Meulen stipend (LvdS), US Public Health Service Grants ES010337, GM086713, and GM126074 (RHT) and by a Grant RVO-VFN64165/2020 from the Czech Ministry of Health (LV).

\section{Author contributions}

L.v.d.S., H.J.V., L.V., R.H.T. and J.W.J. designed experiments and interpreted results. L.v.d.S., A.B., S.d.W., E.M., E.R., A.A.W., R.H., P.V., J.J., D.S., V.W.B., S.C. and A.B.S. performed experiments and analyzed data. L.v.d.S. and J.W.J. wrote the manuscript. 


\section{Competing interests}

The authors declare no competing interests.

\section{Additional information}

Supplementary Information The online version contains supplementary material available at https://doi.org/ 10.1038/s41598-021-90687-5.

Correspondence and requests for materials should be addressed to R.H.T. or J.W.J.

Reprints and permissions information is available at www.nature.com/reprints.

Publisher's note Springer Nature remains neutral with regard to jurisdictional claims in published maps and institutional affiliations.

(c) (1) Open Access This article is licensed under a Creative Commons Attribution 4.0 International License, which permits use, sharing, adaptation, distribution and reproduction in any medium or format, as long as you give appropriate credit to the original author(s) and the source, provide a link to the Creative Commons licence, and indicate if changes were made. The images or other third party material in this article are included in the article's Creative Commons licence, unless indicated otherwise in a credit line to the material. If material is not included in the article's Creative Commons licence and your intended use is not permitted by statutory regulation or exceeds the permitted use, you will need to obtain permission directly from the copyright holder. To view a copy of this licence, visit http://creativecommons.org/licenses/by/4.0/.

(C) The Author(s) 2021 